\title{
QUALIDADE DE VIDA DO FAMILIAR CUIDADOR DE IDOSOS COM ALZHEIMER: CONTRIBUIÇÃO DE UM PROJETO DE EXTENSÃO
}

\author{
Silomar Ilha1 ${ }^{1}$ Cláudia Zamberlan², Maria Helena Gehlen³, Matheus Viero Dias ${ }^{4}$,
} Glaucia Dal Omo Nicola ${ }^{4}$, Dirce Stein Backes ${ }^{5}$

\begin{abstract}
RESUMO: O presente estudo objetivou descrever a percepção dos familiares cuidadores de idosos portadores da doença de Alzheimer, integrantes de um projeto de extensão (AMICA), a respeito da contribuição deste projeto para a melhoria da sua qualidade de vida. Trata-se de uma pesquisa descritiva-exploratória, de caráter qualitativo, desenvolvida por meio de questionário semiestruturado, aplicado entre Abr e maio de 2011, a seis cuidadores/familiares de portadores de Alzheimer. Os dados analisados resultaram nas categorias Qualidade de vida, um bem estar individual e coletivo; Grupo AMICA como promotor da qualidade de vida; e Oportunidade de estabelecer diálogos de saberes resultando em maior compreensão sobre a doença. Neste constructo, o Grupo AMICA tem se mostrado como um importante promotor da qualidade de vida dos familiares cuidadores, os quais reconhecem sua importância. No entanto, torna-se necessário, mais estudos a respeito da qualidade de vida dos familiares cuidadores de idosos portadores da doença de Alzheimer.
\end{abstract}

PALAVRAS-CHAVE: Doença de Alzheimer; Idoso; Qualidade de vida; Enfermagem.

\section{QUALITY OF LIFE FOR FAMILY MEMBERS CARING FOR THE ELDERLY WITH ALZHEIMER'S DISEASE: CONTRIBUTION TO AN EXTENSION PROJECT}

ABSTRACT: This study aimed to describe the perception of family members caring for elderly relatives suffering from Alzheimer's disease, members of an extension project (AMICA), concerning the contribution of the project to the improving of their quality of life. It is descriptive-exploratory research, with a qualitative character, carried out through a semistructured questionnaire, and was applied between April and May of 2011, to six carers/family members of persons with Alzheimer's disease. The data analyzed resulted in the categories Quality of life, individual and collective well-being; the AMICA Group as a promoter of quality of life; and Opportunity to establish dialogs of knowledge resulting in greater comprehension about the illness. The AMICA Group, in this construct, is shown as an important promoter of the quality of life of family carers, who recognize their importance. However, more studies are necessary on the quality of life of family carers for elderly persons suffering from Alzheimer's disease.

KEYWORDS: Alzheimer's disease; Elderly; Quality of life; Nursing.

\section{CUALIDAD DE VIDA DEL FAMILIAR CUIDADOR DE ANCIANO CON ALZHEIMER: CONTRIBUCIÓN DE UN PROYECTO DE EXTENSIÓN}

RESUMEN: El presente estudio tuvo el objetivo de describir la percepción de los familiares cuidadores de ancianos portadores de la enfermedad de Alzheimer, integrantes de un proyecto de extensión (AMICA), acerca de la contribución de este proyecto para mejorar su cualidad de vida. Esta es una investigación descriptiva-exploratoria, de carácter cualitativo, desarrollada por medio de cuestionario semi estructurado, aplicado entre abril y mayo de 2011, a seis cuidadores/familiares de portadores de Alzheimer. Los datos analizados resultaron en las categorías Cualidad de vida, un bienestar individual y colectivo; Grupo AMICA como promotor de la cualidad de vida; y Oportunidad de establecer diálogos de saberes resultando en mayor comprensión sobre la enfermedad. En este constructo, el Grupo AMICA se muestra un importante promotor de la cualidad de vida de los familiares cuidadores, los cuales reconocen su relevancia. Sin embargo, son necesarios más estudios acerca de la cualidad de vida de los familiares cuidadores de ancianos portadores da enfermedad de Alzheimer. PALABRAS CLAVE: Enfermedad de Alzheimer; Anciano; Cualidad de vida; Enfermería.

${ }^{1}$ Enfermeiro. Mestrando em Enfermagem pelo Programa de Pós-Graduação em Enfermagem da Universidade Federal do Rio Grande PPGENF FURG. Bolsista CAPES. Membro do Grupo de Estudos e Pesquisas em Empreendedorismo Social da Enfermagem e Saúde - GEPESES do Centro Universitário Franciscano - UNIFRA.

${ }^{2}$ Enfermeira. Mestre em Enfermagem. Doutoranda em Enfermagem pelo PPGENF FURG. Professora da UNIFRA. Membro do Grupo Interdisciplinar de Pesquisa em Educação e Saúde - GIPES UNIFRA.

${ }^{3}$ Enfermeira. Mestre em Educação. Professora da UNIFRA. Membro do GEPESES.

${ }^{4}$ Enfermeiro. Mestrando em Enfermagem pelo PPGENF FURG. Membro do GEPESES.

${ }^{5}$ Enfermeira. Doutora em Enfermagem. Professora do Curso de Graduação da UNIFRA e do PPGENF FURG. Líder do GEPESES.

Autor correspondente:

Recebido: 19/03/2012

Silomar Ilha Aprovado: 06/06/2012

Universidade Federal do Rio Grande

Rua Maria Noal, 8 - 97020510 - Santa Maria-RS-Brasil

E-mail: silo_sm@hotmail.com

Cogitare Enferm. 2012 Abr/Jun; 17(2):270-6 


\section{INTRODUÇÃO}

A Doença de Alzheimer (DA) foi descrita, pela primeira vez, em 1906 pelo neuropatologista alemão Alois Alzheimer e recebeu essa denominação em 1910 por Kraepelin. E, nessa época, acreditava-se que tal desordem estava restrita a formas graves de demência pré-senil de evolução rápida, com presença de placas senis e de emaranhados neurofibrilares ${ }^{(1)}$. Esta patologia é considerada a forma mais comum de demência entre os idosos; trata-se de uma doença degenerativa que se instala de forma insidiosa e causa declínio progressivo das funções cognitivas ligadas à percepção, à aprendizagem, à memória, ao raciocínio e ao funcionamento psicomotor, bem como ao aparecimento de quadros neuropsiquiátricos com diversas manifestações graves ${ }^{(2)}$.

Estima-se que o número de indivíduos acometidos por DA supere 15 milhões em todo o mundo e sua prevalência vem aumentando de forma significativa, nas diversas faixas etárias ${ }^{(1)}$. De acordo com as recentes análises estatísticas, publicados em 2012, nos Estados Unidos da América existem 5.4 milhões de pessoas com Alzheimer. Destes, 5.2 milhões possuem idade de 65 anos ou mais ${ }^{(3)}$. Acrescenta-se que a cada 68 segundos algum indivíduo na América desenvolve a DA e que no ano de 2050 este intervalo diminuirá para 33 segundos ${ }^{(4)}$.

No Brasil, apesar das estatísticas não serem precisas, estima-se que cerca de 500 mil pessoas sejam acometidas por essa patologia, o que causa impacto social, devido aos custos diretos envolvidos no cuidado com os portadores ${ }^{(1)}$. Acredita-se que atualmente os custos com a DA chegam a 200 bilhões de dólares e que em 2050 os gastos chegarão a 1.1 trilhões de dólares ano ${ }^{(3)}$. Esses fatores colocam esta doença, juntamente com outros tipos de demência, como importante problema de saúde pública em todo o mundo(1).

O Alzheimer leva os indivíduos a um estado de dependência de um cuidador que poderá ser formal ou informal. O cuidador formal é aquele que possui formação específica para o cuidado, geralmente são auxiliares e/ou técnicos em enfermagem que prestam cuidados no domicílio, com remuneração. Enquanto os cuidadores informais são, normalmente, familiares, amigos, vizinhos ou membros de grupos religiosos, sem formação profissional específica e, normalmente, não remunerados ${ }^{(5)}$.

Quando se trata do cuidado ao idoso com DA, na grande maioria das vezes, o cuidado é exercido por um familiar. Essa assistência se dá para que suas necessidades sejam atendidas, já que as dificuldades geradas pela doença se somam às ocasionadas pelo avanço da idade. Dessa forma, o familiar cuidador configura-se como um elemento indispensável no processo saúde-doença, responsabilizando-se por todos os cuidados que envolvem o idoso ${ }^{(1)}$. A escolha do familiar cuidador dar-se-á através de critérios que levem em conta vontade própria de assumir a responsabilidade pelo cuidado diário, disponibilidade de horários, por insistência de outros membros da família, ou até mesmo, por falta de opção, visto ser o único familiar do portador de $\mathrm{DA}^{(5)}$.

Neste processo de cuidado diário, o familiar cuidador, muitas vezes, abdica de suas prioridades em prol do cuidado, fato este que contribui para um declínio na qualidade de vida do cuidador e este declínio, junto ao estresse psicossocial, é influenciado pelo modo como as pessoas (re) organizam suas estratégias de enfrentamento frente à doença ${ }^{(5)}$.

Desse modo, é importante que existam grupos de apoio ao familiar cuidador com vistas à melhoria da sua qualidade de vida. Nessa ótica, a extensão universitária, o ensino e a pesquisa emergem como estratégias consideradas básicas na Universidade, as quais devem ser equivalentes e merecem igualdade em tratamento por parte das instituições de ensino superior. Portanto, este artigo dará ênfase na extensão universitária pela possibilidade de aproximação do meio acadêmico junto à sociedade.

Nesta perspectiva, sustenta-se o Projeto de Extensão intitulado "Assistência Multidisciplinar Integrada aos Cuidadores dos Portadores da Doença de Alzheimer (AMICA)" que possui como objetivo principal prestar assistência ampla aos cuidadores, debatendo assuntos do cotidiano relacionados ao Mal de Alzheimer, favorecendo uma relação de reciprocidade entre os cuidadores, profissionais e estudantes, bem como troca de ideias e de interatividade.

Com base no exposto e em face à doença que vem crescendo significativamente com o aumento da expectativa de vida no Brasil, questiona-se: o Projeto de extensão AMICA tem conseguido alcançar seu objetivo de proporcionar melhoria na qualidade de vida dos familiares cuidadores dos portadores de Alzheimer?

Por meio dos enfoques supracitados, objetivou-se descrever a percepção dos familiares cuidadores de idosos portadores da DA, integrantes de um projeto de extensão, a respeito da contribuição deste projeto para a melhoria da sua qualidade de vida. 


\section{MÉTODO}

Trata-se de uma pesquisa descritiva-exploratória, de caráter qualitativo. Esse tipo de pesquisa objetiva proporcionar uma visão geral sobre determinada situação e pode ser considerada como a primeira etapa de uma pesquisa mais ampla, uma vez que, em decorrência dos seus resultados, podem ser organizados planos estratégicos de ação e, assim, a pesquisa pode contribuir para a mudança da realidade investigada ${ }^{(6)}$.

A pesquisa foi realizada nas dependências do Centro Universitário Franciscano, com familiares cuidadores de idosos portadores de DA que participam do projeto de extensão AMICA. Neste projeto, participam, regularmente, em média 10 familiares cuidadores, porém, não existe um número específico em decorrência da não permanência fixa de muitos familiares, ou seja, alguns participam de encontros, ausentam-se do grupo algum tempo, por motivos diversos e depois retornam.

Como critérios de inclusão, estabeleceram-se: ser familiar cuidador de idoso portador da DA e estar participando assiduamente das reuniões semanais da AMICA. Foram, portanto, excluídos aqueles que são familiares formais, que participam em alguns encontros e ausentam-se do grupo por outro período.

Os dados foram coletados entre os meses de Abr e maio do ano de 2011, por meio de questionário semiestruturado contemplando as seguintes questões norteadoras: "para você o que é qualidade de vida?"; "você acredita que o grupo AMICA contribui para melhoria de sua qualidade de vida? De que maneira?"; "que contribuições os acadêmicos e professores que atuam no grupo agregam aos conhecimentos que você já dispõe por ser cuidador de um idoso portador da doença de Alzheimer?".

Os dados foram analisados e categorizados seguindo a análise de conteúdo de $\operatorname{Bardin}^{(7)}$, a partir de uma pré-análise (organizando o material coletado e sistematizando as ideias através de leitura meticulosa das respostas obtidas na entrevista) e, a seguir, a categorização das unidades de registro, a qual resultou em três categorias.

Foram considerados os preceitos éticos e legais que envolvem a pesquisa com seres humanos, conforme resolução 196/96 do Ministério da Saúde ${ }^{(8)}$. Assim, foi distribuído anteriormente o Termo de Consentimento Livre e Esclarecido para os participantes da pesquisa, sendo este em duas vias, ficando uma em poder do participante e outra em poder do pesquisador.
Manteve-se o anonimato dos depoentes e os mesmos foram identificados pela letra "A" referente AMICA, seguida de um algarismo numérico, conforme ordem de entrevista: (A1, A2, A3...).

A pesquisa obteve a autorização da Coordenadora do projeto de extensão e aprovação pelo Comitê de Ética em Pesquisa do Centro Universitário Franciscano sob o número 435.2010.2.

\section{RESULTADOS}

Atenderam aos critérios de inclusão e aceitaram participar de maneira voluntária, após assinatura do termo de consentimento livre e esclarecido, seis familiares cuidadores de idoso portador de DA.

A partir dos depoimentos dos próprios familiares cuidadores participantes do estudo e da existência de subsídios para a análise, as categorias foram: Qualidade de vida, um bem estar individual e coletivo; Grupo AMICA como promotor da qualidade de vida; Oportunidade de estabelecer diálogos de saberes, resultando em maior compreensão sobre a doença.

Os participantes encontram-se com idades entre 51 e 68 anos, sendo cinco mulheres e um homem. O tempo que estam cuidando diariamente do familiar idoso portador de DA variou entre 4 e 14 anos.

\section{Qualidade de vida, um bem estar individual e coletivo}

Os familiares cuidadores de idosos com Alzheimer, participantes deste estudo, possuem um conceito de qualidade de vida $(\mathrm{QV})$ que permeia lazer, alimentação, atividade física e valores morais individuais e/ou coletivos, como referem nas falas:

É levar uma vida, na medida do possivel, feliz, não sofrendo nenhuma falta dos minimos indispensáveis para sobreviver só, ou em sociedade. (A1)

Estar bem consigo mesma, participar de grupos de convivências, praticar atividade física. Ter uma dieta alimentar de acordo com sua idade e necessidade de saúde. (A2)

É viver com dignidade, estar sempre de bem consigo mesma, com a vida, e cuidar da saúde. (A6)

Alimentação, cuidar bem, viver bem consigo e com os outros, continuar em sociedade. (A3) 
Nos relatos, evidencia-se que os familiares cuidadores possuem uma visão ampliada de QV que vem ao encontro da proposta do grupo. Observa-se que QV faz parte do existir do ser humano como sujeito inserido em uma comunidade, onde englobam, ser feliz, possuir autoestima, cuidar da saúde e dos hábitos alimentares. Contribui para isso, segundo uma respondente, a participação em grupos de convivência. Esta realidade denota, que o AMICA tem conseguido alcançar o objetivo, o qual justifica sua existência que é o de proporcionar - dialogo de saberes entre docentes, discentes e familiares cuidadores - um ambiente participativo e dialógico que proporcione melhoria da QV dos familiares cuidadores.

\section{Grupo AMICA como promotor da qualidade de vida}

Os cuidadores reconhecem a importância da participação no grupo AMICA para o auxílio na QV. Relatam que, por meio do grupo, recebem orientações de forma clara e objetiva, com uma linguagem de fácil assimilação ao senso comum. Por conseguinte, têm-se as falas:

A minha participação nas reuniões do AMICA melhorou minha qualidade, através do auxílio dos acadêmicos nos orientando, no hábito alimentar, na parte de odontologia, enfermagem e outros. (A4)

Ajudam a melhorar minha qualidade de vida através das orientações claras, objetivas e de fácil assimilação. (A2)

Através das minhas participações nas reuniões, como auxilio que os acadêmicos me dão através das orientações nos hábitos alimentares, na parte da odontologia, enfermagem e outros. (A5)

Esclarecendo minhas dúvidas, aprendo muita coisa no grupo. A conviver com pessoas na mesma situação que eu e a me preparar para a vida futura com o portador, cuidando mais de minha saúde, melhorando, consequentemente, minha qualidade de vida. (A1)

Como se evidencia nos relatos, os familiares reconhecem a participação dos graduandos dos cursos da saúde no que se refere ao diálogo que resulta em orientações e maior compreensão sobre a importância do autocuidado, contribuindo em uma melhoria da
QV. Isso corrobora com o fato de que o graduando, independente da área de atuação, consegue estabelecer por meio da comunicação, um viés transformador.

As ações educativas promovidas pelo grupo de profissionais e alunos constituem práticas interdisciplinares no ensino, são realizadas de maneira dialógica com os saberes e culturas, permitindo ampliar a capacidade de conhecer a realidade e intervir com responsabilidade sobre ela, na garantia de vida com qualidade a todos, principalmente, ao familiar cuidador.

\section{Oportunidade de estabelecer diálogos de saberes, resultando em maior compreensão sobre a doença}

Observa-se que, no grupo, ocorre a comunicação de tripla via, caracterizada pelo contato entre os familiares cuidadores, profissionais e acadêmicos das áreas da saúde, integrantes do projeto. Assim, o saber dos graduandos e dos profissionais de saúde une-se aos adquiridos pelos familiares no processo de cuidar. A seguir, observam-se as falas:

É uma troca de informação. Já aprendi como preparar uma alimentação saudável, como tratar a higiene bucal, como entender o doente, maneiras de alimentá-los, o efeito dos medicamentos, a importância da movimentação, a importância do banho, e muito mais. (A2)

A partir das orientações fornecidas no grupo entendemos que devemos cuidar do nosso doente de maneira segura e carinhosa, mas não podemos esquecer dos nossos próprios cuidados. (A5)

Através das trocas de informações entre os acadêmicos e os professores, agregamos mais orientações de como lidar com os doentes, por exemplo: como fazer a troca de roupa, a maneira de levantar uma pessoa que já não se ajuda mais, virá-la na cama e mais outras orientações. (A4)

Os acadêmicos e professores me ajudaram bastante de como o portador se comporta e como cuidar e entendelo, o porquê ele age daquele jeito. (A3)

As falas traduzem o que se pretende com este grupo, quanto à socialização de saberes entre profissionais, acadêmicos e familiares, contribuindo com a comunidade e com o meio acadêmico, em prol de uma melhoria da QV.

Por meio do AMICA, a equipe possui a oportuni- 
dade de proporcionar orientações acerca do cuidado prestado, bem como do autocuidado, visto que a doença apresenta-se de diferentes maneiras, e o cuidador não poderá deixar de cuidar-se em prol de prestar o cuidado. Em especial, oportuniza-se definir alguns conceitos que trazem dúvidas aos cuidadores, os quais podem aproveitar a oportunidade para saná-las.

\section{DISCUSSÃO}

A QV pode ser entendida como uma percepção eminentemente humana que abarca diversos significados derivados de experiências, valores e conhecimentos individuais e coletivos ${ }^{(9)}$. Atualmente, a definição mais divulgada e conhecida é a do grupo da Organização Mundial da Saúde (OMS) que a descreve como a percepção que o indivíduo tem sobre sua posição na vida, no contexto da cultura e sistemas de valores nos quais vive e em relação aos seus objetivos, expectativas, padrões e preocupações ${ }^{(10)}$.

Pode-se afirmar que o conceito de QV é amplo e inclui uma variedade de dimensões que não se limitam às condições de saúde, controle de sintomas e intervenções médicas ao portador. Além dos indicadores objetivos, devem ser consideradas algumas dimensões menos tangíveis, como: sentido de segurança, dignidade pessoal, oportunidades de atingir objetivos, satisfação com a vida, alegria e sentido positivo de si $^{(11)}$.

No que se refere à QV dos familiares cuidadores, alguns autores têm se dedicado a investigar os efeitos estressores e seus impactos. Ser cuidador demanda tempo, amor, respeito, carinho, energia, paciência, esforço e boa vontade, uma vez que as graduais perdas cognitivas, mudanças comportamentais, emocionais e até de personalidade do idoso exigem uma grande capacidade de adaptação para que haja um convívio satisfatório ${ }^{(12)}$.

Quando se trabalha em grupo, se tem a oportunidade de estimular os participantes a encontrar estratégias coletivas de enfrentamento dos problemas vivenciados, onde cada indivíduo tem a possibilidade de expressar seus sentimentos e pensamentos, dar opiniões, expor seu ponto de vista ou permanecer em silêncio, de maneira que haja o compartilhamento de conhecimentos advindos das experiências de cada um. É, sobretudo, por meio das trocas, que as pessoas vão se beneficiar, encontrando respostas para a maioria de suas dúvidas, efetivando-se gradativamente a educação em saúde por meio dos grupos ${ }^{(13)}$.

Denota-se, assim, a importância desta vivência para os graduandos dos cursos da saúde quais sejam de clínica ou outros saberes, pois o saber acadêmico, contextualizado com os dos profissionais e dos familiares, permite dizer que as atividades realizadas de forma interdisciplinar preconizam uma tecnologia menos dura do que aparelhos e equipamentos utilizados na prática ${ }^{(14)}$. Ratificando essa ideia, percebe-se que praticar temáticas voltadas para educação, tanto de profissionais quanto de estudantes, proporcionou um olhar singular à pessoa com DA e, à sua família, um atendimento holístico que auxilia na promoção da saúde, a fim de melhorar a QV.

A DA acarreta profundas alterações no portador, bem como no contexto familiar em que está inserida, trazendo abalo e sobrecarga emocional, principalmente no que tange à sintomatologia de perda da memória ${ }^{(15)}$. $\mathrm{O}$ familiar cuidador configura-se como um elemento indispensável no processo, responsabilizando-se por todos os cuidados que envolvem o idoso. Porém, esse indivíduo possui uma concepção de saúde e doença que está diretamente associada à forma como ele, ao longo de sua história de vida, foi apropriando-se da natureza para mudá-la, buscando atender às suas necessidades, ou seja, não dispõe de formação específica e conhecimentos científicos que respaldem sua prática de cuidados ${ }^{(15)}$.

Neste constructo, levando-se em conta que a doença, além de manifestar-se por perdas cognitivas, provoca distúrbios de comportamento e afeto, e que o familiar ao assumir a responsabilidade do cuidado, deve possuir condições seguras para fazê-lo, percebese a importância das orientações ao familiar cuidador para trabalhar as questões que se tornam cotidianas quando se possui um membro familiar com DA.

Assim, há a necessidade do profissional conhecer e compreender a realidade da vida diária do cuidador do idoso portador de Alzheimer, recuperando valores de vida, condições sociais e maneiras de enfrentar os problemas. Isso exige dos enfermeiros, bem como dos demais profissionais da área da saúde, conhecimento, adequação e abordagem que inclua a família no planejamento das ações de cuidado(15).

Na perspectiva de trocas e de apoio mútuo, tem-se a conformação do grupo, que emerge como caminho para a efetivação das trocas de que os cuidadores tanto precisam para qualificação de sua assistência. Portanto, pode-se afirmar que a prática de grupo representa uma forma de promover o aprendizado no que diz respeito à educação e à saúde, além do que a vivência em grupo possibilita a inclusão e a valorização, tendo 
em vista que muitos indivíduos procuram, através de discussões e experiências coletivas, subsídios para o enfrentamento de problemas de saúde ${ }^{(16)}$.

\section{CONCLUSÕES}

As dificuldades encontradas pelo idoso portador da DA e seu familiar cuidador são diversas. Desta forma, torna-se importante trabalhar com familiares utilizando novos métodos que contemplem não apenas o amenizar da doença, mas sim, a efetiva atuação em um contexto interdisciplinar, que vise maior autonomia, qualidade de vida e bem-estar, uma vez que nenhuma abordagem isolada na área da saúde poderá dar conta da complexidade.

Diante disso, pode-se dizer que há urgência na criação de novos serviços que atendam a esta clientela, e assim justifica-se a importância dos projetos de extensão com vistas na melhoria da QV do familiar cuidador. Por meio da extensão universitária a Universidade interage com a comunidade na qual está inserida, tendo subsídios para posteriores pesquisas, auxiliando na formação de profissionais, já que aproxima o saber científico de realidades encontradas na prática.

Neste constructo, o AMICA tem se mostrado como um importante promotor da QV dos familiares cuidadores, os quais reconhecem a importância do grupo. No entanto, torna-se necessário, mais estudos voltados a resultados conquistados por meio de projetos de extensão universitária, bem como a respeito da QV do familiares cuidadores de idosos portadores da DA.

A sociedade, por sua vez, precisa entender que a situação vivenciada pelo idoso com Alzheimer não é apenas um problema privado, mas também uma questão de saúde pública, que demanda grande investimento financeiro em programas e políticas sociais de atenção e promoção da QV dos idosos e de suas famílias.

\section{REFERÊNCIAS}

1. Freitas ICC, Paula KCC, Soares JL, Parente ACM. Convivendo com o portador de Alzheimer: perspectivas do familiar cuidador. Rev Bras Enferm. 2008;61(4):508-13.

2. Valim MD, Damasceno DD, Abi-acl LC, Garcia F, Fava SMCL. A doença de Alzheimer na visão do cuidador: um estudo de caso. Rev. Eletr. Enf. [Internet] 2010;12(3):528-34.

3. Alzheimer's Association, 2012. Alzheimer's disease facts and figures. Alzheimer's dementia. 2012;8(2):131-8.

4. Corrada MM, Brookmeyer R, Paganini-Hill A, Berlau $\mathrm{D}$, Kawas CH. Dementia incidence continues to increase with age in the oldest old: The $90+$ study. Ann Neurol. 2010;67(1):114-21.

5. Bulla LC, Martins RR, Valencio G. O convívio do idoso com Alzheimer com sua família: Qualidade de vida e suporte social. In: Bulla, LC, Argimon IIL, organizadores. Convivendo com o familiar idoso. Porto Alegre: EDPUCRS, 2009.

6. Cansonieri AM. Metodologia da pesquisa qualitativa na saúde. $1^{\mathrm{a}}$ ed. Rio de Janeiro, Vozes; 2010.

7. Bardin L. Análise de conteúdo. $4^{\mathrm{a}}$ ed. Lisboa: Edições; 2009.

8. Ministério da Saúde (BR). Conselho Nacional de Saúde. Diretrizes e normas regulamentadoras de pesquisa envolvendo seres humanos. Resolução n. 196, de 10 de outubro de 1996. Brasília; 1996.

9. Borghi AC, Sassá AH, Matos PCB, Decesaro MN, Marcon SS. Qualidade de vida de idosos com doença de Alzheimer e de seus cuidadores. Rev. Gaúcha Enferm. 2011;32(4):751-8.

10. Campos MO, Neto JFR. Qualidade de vida, um instrumento para promoção da saúde. Rev. baiana de saude publica. 2008;32(2):232-40.

11. Pinto MF, Barbosa DA, Ferreti CEL, Souza LF, Fram DS, Belasco AGS. Qualidade de vida de cuidadores de idosos com doença de Alzheimer. Acta Paul. Enferm. 2009;22(5):652-7.

12. Celich KLS, Batistella M. Ser cuidador familiar do portador de doença de Alzheimer: vivências e sentimentos desvelados. Cogitare enferm. 2007; 12(2):143-9.

13. Arruda MC, Alvarez AM, Gonçalves LHT. O familiar cuidador de portador de doença de Alzheimer participante de um grupo de ajuda mútua. Cienc. cuid. saude. 2008;7(3):339-45.

14. Lopes WO, Saupe R, Massaroli A. Visita domiciliar: tecnologia para o cuidado, o ensino e a pesquisa. Cienc. cuid. saude. 2008;7(2):241-7.

15. Inouye K, Pedrazzani ES, Pavarini SCI, Toyoda CY. Perceived quality of life of elderly patients with dementia and family caregivers: evaluation and correlation. Rev. 
Latino-Am. Enfermagem. 2009;17(2):187-93.

16. Dall'Agnol CM, Resta DG, Zanatta E, Schrank G, Maffacciolli R. O trabalho com grupos como instância de aprendizagem em saúde. Rev. Gaúcha Enferm. 2007;28(1):21-6. 